\title{
The cognitive and psychosocial effects of auditory training and hearing aids in adults with hearing loss
}

\author{
Joanna Nkyekyer ${ }^{\prime}$ \\ Denny Meyer ${ }^{2}$ \\ Andrew Pipingas ${ }^{3}$ \\ Nicholas S Reed ${ }^{4}$
}

'Australian Research Council Training Centre in Biodevices, Swinburne University of Technology, Melbourne, VIC, Australia; ${ }^{2}$ Department of Statistics Data Science and Epidemiology, Swinburne University of Technology, Melbourne, VIC, Australia; ${ }^{3}$ Center for Human Psychopharmacology, Swinburne University of Technology, Melbourne, VIC, Australia; ${ }^{4}$ Cochlear Center for Hearing and Public Health, Johns Hopkins University Bloomberg School of Public Health, Baltimore, MD, USA

Correspondence: Joanna Nkyekyer Australian Research Council Training Centre in Biodevices, Swinburne University of Technology, PO Box 218, Hawthorn, VIC 3I22, Australia

Tel +6I 422733 I 57

Email jnkyekyer@swin.edu.au
This article was published in the following Dove Medical Press journal: Clinical Interventions in Aging

\begin{abstract}
Purpose: Our study assessed the efficacy of the simultaneous use of hearing aids and auditory training for improving cognition and psychosocial function in adults with hearing loss, and the relationships between hearing loss, speech perception and cognition.
\end{abstract}

Participants and methods: A 40-person (aged 50-90 years) pilot study in Melbourne, Australia, was conducted. Participants with hearing impairment completed the Geriatric Depression Scale-Short Form, questions about social activity participation, a wide range of cognitive tasks and a speech perception test at baseline, 3 and 6 months. Participants underwent auditory training for 6 months and used hearing aids for 3 months.

Results: Correlations and structural equation modeling suggested that several cognitive domains were associated with speech perception at baseline, but only the Incongruent Stroop cognition measure was associated with hearing loss. Hearing aid use reduced problems with communication, but there were no significant improvements in speech perception, social interaction or cognition. The effect of hearing aids and auditory training for improving depressive symptoms was significant with a moderate to large effect size (Cohen's $d=0.87$ ).

Conclusion: The small sample size and a relatively high rate of attrition meant that this study was underpowered. However, baseline results suggested relationships between hearing loss, speech perception and cognition, and the hearing intervention provided evidence of reduced depressive symptoms. A full-scale, randomized hearing loss intervention and a longer neuroimaging study with cognitive outcomes measured in the short term as well as after several years of hearing aid use are needed.

Keywords: cognition, depression, hearing loss intervention, speech perception

\section{Introduction}

Hearing loss is a highly prevalent chronic disability among older adults. Two-thirds of adults aged 70 years or older have clinically meaningful hearing loss. ${ }^{1}$ Age-related hearing loss is associated with permanent damage at the cellular level of the auditory system. This is not correctable by either surgical or pharmacologic interventions.

Recent studies have demonstrated an association between age-related hearing loss and difficulties in understanding speech, ${ }^{2}$ cognitive decline and incident dementia. ${ }^{3}$ The mechanisms by which hearing loss may impact cognition are thought to be associated with increased cognitive load, changes in brain structure, decreased social engagement and depression. ${ }^{4}$ Research suggests that hearing loss increases the cognitive load on brain activity by diverting cognitive resources to process the degraded auditory signal, at the expense of other cognitive processes such as working memory. ${ }^{5}$ Studies have shown that among adults with hearing impairments, a greater number of depressive 
symptoms are associated with cognitive and concentration disorders, which may be improved by hearing aids. ${ }^{6}$ Better understanding of the etiology behind the connection between hearing loss and cognitive decline could help lead to interventions that preserve cognitive function in hearing loss patients, and hence the need for studies such as this.

The current gold standard in addressing hearing loss is amplification by hearing aids, which involves restoring the audibility of sounds in order to improve speech perception. ${ }^{7}$ Despite known consequences of hearing loss and significant advances in hearing aid technology, only $14 \%$ of US adults over the age of 50 years who might benefit from hearing aids actually use them. ${ }^{8}$ Lack of access, stigmatization, difficulty in managing hearing aids and/or an underestimation of hearing aid benefit may contribute to low adoption rates for hearing aids. ${ }^{9}$ Also, hearing aids alone often do not overcome all adverse listening environments and first-time hearing aid users require substantial counseling and auditory training in order to ensure that they make the best use of their hearing aids. ${ }^{10}$ In addition, few studies have provided methodologically rigorous evidence that hearing aids improve health outcomes such as cognition in older adults. ${ }^{11}$

Auditory training is the use of instruction, drill or practice, designed to increase the amount of information that hearing contributes to a person's total perception. ${ }^{12}$ Although studies have shown that auditory training results in improvements in the understanding of speech-in-noise, it is not commonly recommended to adults with hearing loss. These studies have shown that auditory training improves communication outcomes for first-time hearing aid users by optimizing acclimatization to the new auditory cues provided by hearing aids in adverse listening conditions. ${ }^{13,14}$

However, despite this evidence, auditory training is rarely provided in conjunction with hearing aid fitting, possibly due to the lack of reimbursement for providers, and no previous studies have investigated the effect of hearing aids in conjunction with auditory training on health outcomes such as cognition. The aim of this study was to examine the efficacy of the simultaneous use of hearing aids and individualized face-to-face auditory training for improving cognition, social interaction and depressive symptoms for first-time hearing aid users. Exploratory analyses also investigated the mediating role of a web-based speech perception test (SPT).

\section{Participants and methods Design}

This study was a randomized crossover pilot study of 40 men and women with mild-to-moderate sensorineural hearing loss (SNHL). Participants were recruited from eight independent living retirement facilities and surrounding communities across Melbourne, Australia. The complete study protocol is described elsewhere. ${ }^{15}$

Study eligibility criteria specified adults aged between 50 and 90 years, with bilateral symmetric SNHL in the mild to moderate range based on a pure tone average of $25 \mathrm{~dB}$ hearing loss (HL) to $70 \mathrm{~dB}$ HL. Also, participants needed to be proficient in English, to have never worn hearing aids before and to express willingness to wear hearing aids for 3 months and attend weekly auditory training for 6 months. Exclusion criteria included suspected cognitive impairment as defined by a score $\leq 24$ on the mini-mental state examination questionnaire, and uncorrected visual impairment and/or color blindness as required by the Swinburne University Computerized Cognitive Assessment Battery (SUCCAB) test. This study was conducted in compliance with the Swinburne's Human Research Ethics Committee requirements (SHR Project 2016/159) and was registered at ClinicalTrials.gov as NCT03112850. The study was performed in accordance with the principles stated in the Declaration of Helsinki. ${ }^{16}$ All participants provided written informed consent prior to commencement of the study.

Enrolled participants completed an individualized inperson auditory training program for a period of 6 months and were randomly allocated into two groups of equal size ( $n=20$ per group) as follows:

- Participants fit with hearing aids only for the first 3 months of the auditory training program were assigned to Group A.

- Participants fit with hearing aids only for the last 3 months of the auditory training program were assigned to Group B.

The hearing intervention included intensive weekly auditory training for a period of 6 months with the wearing of hearing aids for either the first 3 months or the last 3 months of the auditory training program. Participants were tested at baseline and at 3 and 6 months in terms of cognition, depressive symptoms, social interaction and hearing satisfaction.

\section{Intervention}

\section{Hearing aid fitting}

Participants were provided with bilateral Blamey Saunders LOF (originally known as Liberty Open-Fit) hearing aids by the study audiologist. Hearing aids were adjusted and customized according to the Blamey and Saunders best-practice protocol. The study audiologist provided an oral explanation on how to use the hearing aids, and a step-by-step guide on 
hearing aid use was provided to the participants as take-home reading. To increase hearing aid compliance and to manage the expectations as needed, counseling support was provided to the participants at weekly auditory training sessions. An automatic Internet-based data logging function installed in the hearing aids was used to assess hours of hearing aid use over the 3-month period of hearing aid use.

\section{Auditory training}

Study participants received weekly face-to-face auditory training sessions of Continuous Discourse Speech Tracking. ${ }^{17}$ In this process, a trained study member read a novel/short story and the participant was instructed to repeat back verbatim each sentence or phrase. If the repetition was correct, the researcher articulated the next phrase or sentence. If the repetition was incorrect, the researcher repeated the phrase or sentence, or a portion of it, or used other repair strategies, until the sentence or phrase was correctly repeated in its entirety. Training materials were tailored to the personal interests of participants. Each training session lasted for 15 minutes.

\section{Outcome measures}

\section{Blamey Saunders SPT}

In addition to the standard audiogram, we used a web-based SPT to measure hearing loss, with and without hearing aids. The SPT is a monosyllabic word test that generates a display of information transmission for 50 vowels and 100 consonants in order to characterize the shape and degree of hearing loss, analogously to an audiogram. ${ }^{18,19}$ The SPT is similar to consonant-vowel nucleus-consonant word tests that has been used previously for various speech recognition tests. ${ }^{20,21}$ The SPT consists of the following: 50 consonantvowel-consonant words designed for use in any reasonably quiet environment, presented at a "comfortable level" of 65 dB; Australian English spoken by a native Australian female speaker; one list of words (in a random order) randomly chosen from 32 phonetically balanced lists of words; responses typed by the listener; automatic analysis and reporting of word consonant and vowel scores. The SPT score used for this study was the phoneme score - the total number of correctly identified vowels and consonants. Higher SPT scores suggest better hearing.

Participants completed the SPT without hearing aids at baseline, after 3 months and then at 6 months. The SPT was also performed with hearing aids immediately after the participants were fitted with hearing aids for the first time, and at the end of 3 months of auditory training while wearing a hearing aid.

\section{Subjective assessments of hearing}

For subjective assessments of hearing (with or without hearing aids), the Abbreviated Profile of Hearing Aid Benefit (APHAB) was used. Participants answered the APHAB at baseline, after 3 months and after 6 months, and we used the $\mathrm{APHAB}^{22}$ to assess the following different listening situations in daily life, without hearing aids and with hearing aids when relevant: Ease of Communication (EC), Background Noise (BN), Reverberation of sound (RV), such as listening to sounds across a large room and Aversiveness of sound (AV), which measures uncomfortable loudness of background sounds such as traffic and alarm bells. We expected that participants with worse hearing would score higher on all four subscales at baseline, but would improve due to hearing and auditory interventions.

Using a 7-point scale, respondents indicated how often the statement was true for them. Each point on the scale provided a descriptor and an associated percentage of time, with higher scores indicating more of a problem:

\begin{tabular}{|l|l|l|}
\hline A & Always & $99 \%$ \\
\hline B & Almost always & $87 \%$ \\
\hline C & Generally & $75 \%$ \\
\hline D & Half the time & $50 \%$ \\
\hline E & Occasionally & $25 \%$ \\
\hline F & Seldom & $12 \%$ \\
\hline G & Never & $1 \%$ \\
\hline
\end{tabular}

\section{Speech tracking rates from auditory training}

To analyze our speech tracking rates from the auditory training, we used the following "learning and forgetting" mathematical model as described by Blamey and Alcantara: ${ }^{12}$

$$
R(t)=\frac{1-\exp (-f(t-T))}{L / f}
$$

where

- $R(t)=$ tracking rate in words per minute at time $t$;

- $L=$ learning rate per week, that is, the increase in speech tracking rate after 1 week of auditory training;

- $f=$ forgetting rate per week, that is, the reduction in speech tracking rate in between speech tracking sessions;

- $t$ is time in weeks, $t>0$ and

- $T$ is a constant.

The parameters $L, f$ and $T$ were estimated for each group over both 3-month periods separately. The $L$ and $f$ parameter estimates are valid measures of learning and forgetting cognitive processes, which may be affected by the use of hearing aids and/or auditory training. 
Research in auditory training has shown that the amount of training, the amount of learning, the generalization of skills (whether auditory training will improve communication in real-life situations as well as under artificial test conditions) and the degree of retention of skills are all interrelated. ${ }^{12}$ Therefore, a mathematical formula was helpful for tracking improvements in auditory training over time.

\section{Cognitive assessments - SUCCAB}

The SUCCAB assessed eight cognitive domains (Simple and Complex Reaction Times, Immediate and Delayed Recognition, Congruent and Incongruent Stroop, Spatial Working Memory and Contextual Memory) ${ }^{23}$ Computerized measures provided consistency in measurement across participants and a more automated approach in analysis.

A performance score for each task was calculated as the ratio of accuracy and reaction time. This approach took into account variations in accuracy and response time, allowing for speed vs accuracy trade-offs in performance. Some of these tests require the identification of colors, hence the need for color blindness as an exclusion criterion for this trial. We expected that participants with better hearing would have better cognition scores at baseline.

\section{Psychosocial assessments (depressive symptoms and social interaction)}

The short form of the Geriatric Depression Scale (GDS) ${ }^{24}$ was used for measuring depressive symptoms. The GDS has been found to be a reliable and valid measure of depressive symptom $\mathrm{s}^{25}$ and to be highly correlated with other measures of such symptoms. The GDS was designed for older adults. Items are scored dichotomously (respondents answer "Yes" or "No" to five items). Items assess non-somatic aspects of depression, thus allowing for discrimination between respondents with depressive symptom and those with medical problems. Higher scores (a score above 5) are suggestive of depression.

We used the Berkman-Syme Social Network Index ${ }^{26}$ to assess participants' social interaction and connections with families and friends. Twelve types of social relationships were assessed, namely, relationships with the spouse, parents, parents-in-law, children, other close family members, close neighbors, friends, workmates, schoolmates, fellow volunteers, members of groups without religious affiliation and religious groups. We expected that people with better hearing will have higher Berkman-Syme Social Network Index scores at baseline.

\section{Statistical analyses}

The two participant groups were compared in terms of demographic factors and baseline values using chi-squared and independent samples $t$-tests, with tests for correlations between the outcome measures and age at baseline. Square root transformations were applied where necessary.

Second, structural equation modeling was used to examine the relationships among hearing loss, speech perception and cognition. Fitting the structural model using maximum likelihood estimation, goodness of fit was evaluated using a chi-squared goodness of fit statistic.

Thirdly, separately for each group, we reported the objective and perceived benefit of hearing aids for the 3-month period of hearing aid usage, using paired $t$-tests to evaluate the significance of any improvement in terms of hearing satisfaction and SPT.

Fourthly, we compared speech tracking learning curves for groups A and B separately for the first 3 months and the last 3 months, in order to monitor the effects of the auditory training over time, with and without hearing aids.

Finally, a mixed model analysis was performed for our crossover design to determine whether hearing aids had any significant effect on cognition and psychosocial function over and above auditory training, and to establish the significance of any period or carryover effects.

\section{Results \\ Participants}

From December 2016 to March 2017, 84 individuals were screened for eligibility. Of these, 54 (64.3\%) participants were recruited from independent living retirement villages, while 30 (35.7\%) participants were from surrounding communities. After screening, 40 participants were found to be ineligible and 4 did not want to either wear hearing aids or undergo auditory training and, therefore, declined to participate in the study. The remaining 40 participants were randomly allocated - 20 to Group A and 20 to Group B.

Out of the 40 participants, 21 (52.5\%) had mild symmetric SNHL (between 21 and $40 \mathrm{~dB}$ ) and $19(47.5 \%$ ) had moderate symmetric SNHL (between 41 and $70 \mathrm{~dB}$ ). There were no significant differences between the groups at baseline; however, moderate to large effect sizes (Cohen's $d$ ) are indicated for Contextual Recognition Memory and Aversiveness of sound (see Table S1 for baseline characteristics).

\section{Effects of hearing loss on cognition}

Table 1 shows the relationship between all the cognitive and psychosocial measures, the APHAB measures (without 
Table I Pearson's correlations for baseline results without hearing aids

\begin{tabular}{|l|l|l|l|}
\hline Outcome measures & Age (years) & Hearing loss (PTA) & SPT \\
\hline Age (years) & 1.000 & 0.278 & $-0.443^{* *}$ \\
\hline Hearing loss (PTA) & 0.278 & 1.000 & $-0.695^{* *}$ \\
\hline SPT & $-0.443^{* *}$ & $-0.695^{* *}$ & 1.000 \\
\hline Cognition: Simple Reaction Time & $-0.369^{*}$ & -0.301 & $0.338^{*}$ \\
\hline Cognition: Complex Reaction Time & $-0.390^{*}$ & -0.114 & 0.223 \\
\hline Cognition: Immediate Recognition Memory & $-0.555^{* *}$ & -0.145 & 0.300 \\
\hline Cognition: Delayed Recognition Memory & $-0.500^{* *}$ & -0.040 & 0.279 \\
\hline Cognition: Stroop Congruent & $-0.400^{*}$ & -0.219 & $0.492^{* *}$ \\
\hline Cognition: Stroop Incongruent & -0.079 & $-0.323^{*}$ & 0.265 \\
\hline Cognition: Spatial Working Memory & $-0.325^{*}$ & -0.153 & $0.393^{*}$ \\
\hline Cognition: Contextual Recognition Memory & $-0.522^{* *}$ & -0.083 & $0.405^{* *}$ \\
\hline APHAB: SQRT EC & 0.129 & $0.404^{* *}$ & $-0.578^{* *}$ \\
\hline APHAB: RV & 0.122 & 0.297 & $-0.332^{*}$ \\
\hline APHAB: BN & -0.121 & 0.226 & $-0.317^{*}$ \\
\hline APHAB: SQRT AV & 0.081 & -0.121 & -0.170 \\
\hline SQRT depression & 0.014 & 0.057 & -0.019 \\
\hline Social interaction & $-0.352^{*}$ & -0.095 & 0.238 \\
\hline
\end{tabular}

Notes: ${ }^{*}$ Correlation is significant at the 0.05 level (two-tailed). ${ }^{* *}$ Correlation is significant at the 0.01 level (two-tailed).

Abbreviations: APHAB, Abbreviated Profile of Hearing Aid Benefit; AV, Aversiveness of sound; BN, Background Noise; EC, Ease of Communication; PTA, pure tone average; RV, Reverberation of sound; SPT, speech perception test; SQRT, square root transformation.

hearing aids), with age, hearing loss and speech perception (without hearing aids), demonstrating several significant correlations with SPT results at baseline. In particular, as expected, there was a strong negative correlation between SPT and hearing loss and significant negative correlations of moderate size between age and cognition.

Also, as expected, there were significant, but weaker negative correlations between SPT and the APHAB hearing problems. Contrary to expectation, there was only one significant correlation between hearing loss and cognition (Incongruent Stroop) and one significant correlation between hearing loss and problems identified with hearing (Ease of Communication). No significant correlations were observed for social interaction or depression.

Due to the negative effect of hearing loss on speech perception, we further investigated the mediating role of speech perception on cognition at baseline.

Figure 1 illustrates the association between hearing loss, unaided speech perception and cognition, providing a good fit for the data $\left(\chi^{2}=40.67, d f=35, P=0.235\right)$. In this model, cognition is measured as a latent variable and, although it is assumed that SPT and cognition are correlated, no assumption about the direction of this relationship is made.

\section{Effects of hearing aid use and auditory training on cognition}

\section{Assessment of outcome of hearing aids}

The average daily hearing aid use for Group A and Group B as measured through objective data logged by the hearing aid for the 3-month period (when hearing aids were first fitted) was 4.6 and 5.25 hours, respectively. However, there was a high proportion of missing data for this variable, making these results unreliable.

SPTs were performed immediately after hearing aid fitting and again at the end of 3 months of auditory training and hearing aid usage (\#). The results showed no significant change for Group A or Group B (see Table 2). However, when we compared SPT results without wearing hearing aids before and after 3 months of auditory training and hearing aid usage (@), there was a significant improvement for Group A and nearly a significant improvement for Group B.

Finally, we compared the SPT results without hearing aids and immediately after hearing aid fitting (see Table S2 for the outcome of perceived hearing aid benefit). Interestingly, there was a significant immediate improvement when hearing aids were first fitted for Group A, which was nearly significant for Group B. However, when the hearing aids were 


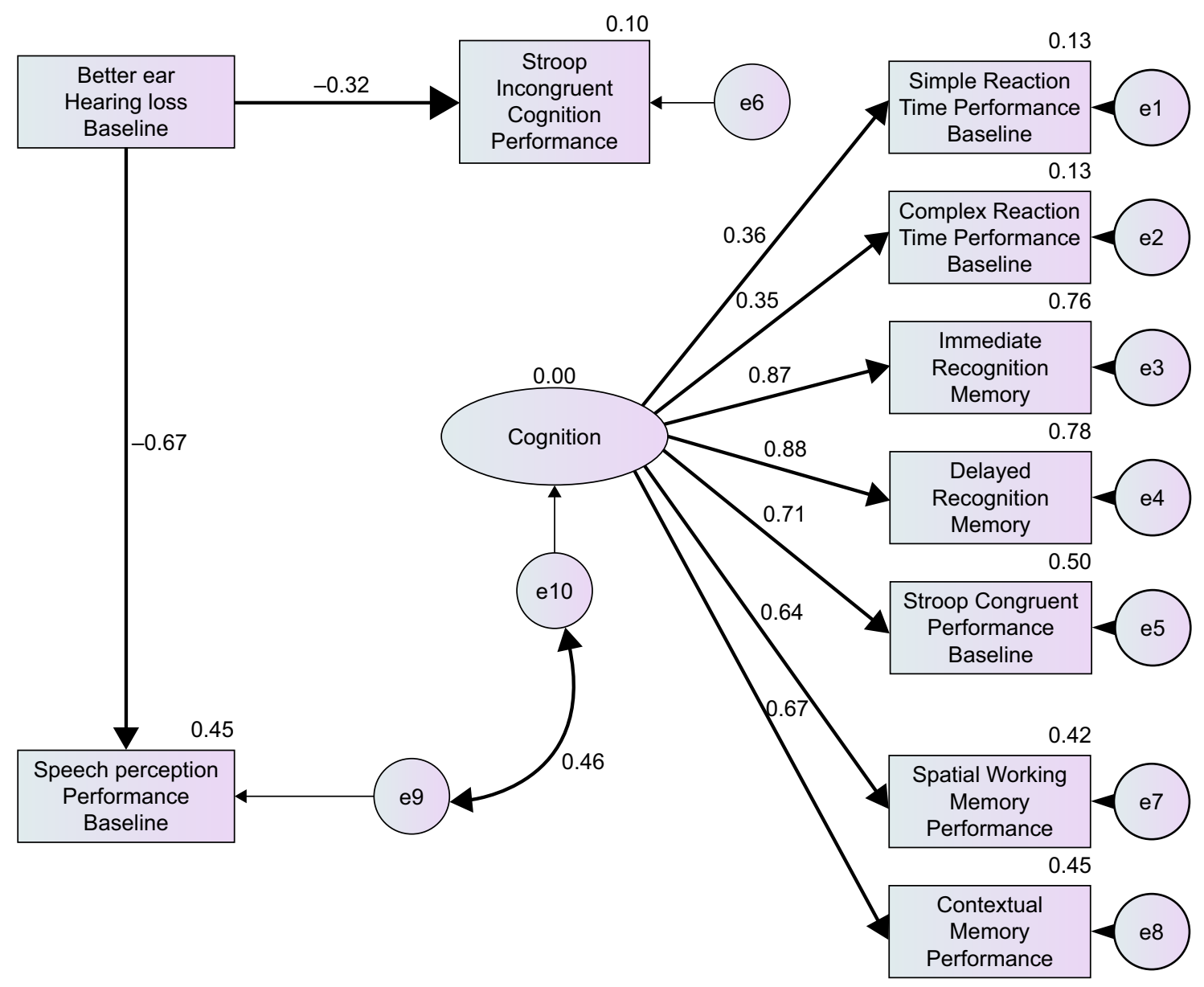

Figure I Structural equation model with $R^{2}$ values and standardized path coefficients with significant $(P<0.05)$ paths in bold.

finally removed, at 3 months for Group A and at 6 months for Group B, the SPT results showed no significant difference for either group with and without hearing aids. The unaided SPT scores increased during the intervening auditory training period, although not significantly $(P=0.543$ for Group A and $P=0.064$ for Group B), but the aided SPT scores remained fairly constant.

\section{Effectiveness of auditory training}

Fitted learning curves (with and without hearing aids) from the speech tracking are displayed in Figure 2 (see Table S3 for the estimated parameters from auditory training). It was expected that it would be easier to learn to do speech tracking when participants used hearing aids than when they did not, and that using hearing aids during the week would mean

Table 2 Outcome of perceived hearing aid benefit

\begin{tabular}{|c|c|c|c|c|c|c|c|c|}
\hline \multirow[t]{2}{*}{ Domain } & \multicolumn{4}{|c|}{$\begin{array}{l}\text { Group A, } n=19 \text {, } \\
\text { mean (SD) }\end{array}$} & \multicolumn{4}{|c|}{$\begin{array}{l}\text { Group B, } n=14 \text {, } \\
\text { mean (SD) }\end{array}$} \\
\hline & Baseline & 3 months & $t$-value & $P$-value & 3 months & 6 months & $t$-value & $P$-value \\
\hline $\mathrm{EC}$ & $27.2(18.7)$ & I $7.0(\mid 7.0)$ & 2.809 & 0.012 & I8.4 (I5.3) & I2.8(I0.5) & 1.298 & 0.217 \\
\hline $\mathrm{RV}$ & $36.0(14.0)$ & $29.3(16.1)$ & 2.614 & 0.017 & $30.3(13.6)$ & 20.0 (II.7) & 3.187 & 0.007 \\
\hline $\mathrm{BN}$ & $36.6(13.9)$ & $24.7(16.5)$ & 2.926 & 0.009 & $31.3(15.0)$ & $26.1(13.3)$ & 2.062 & 0.060 \\
\hline AV & 36.4 (22.7) & $48.1(23.5)$ & 2.275 & 0.035 & |4.3 (I I.8) & $37.2(29.3)$ & 3.357 & 0.005 \\
\hline SPT\# & I25.I (I5.7) & I24.5 (I7.2) & 0.459 & 0.652 & $124.0(16.7)$ & I22.9 (I7.8) & 0.473 & 0.644 \\
\hline SPT@ & $120.0(18.4)$ & I 24.5 (I7.2) & 2.319 & 0.032 & $119.0(18.6)$ & I22.9(17.8) & 2.025 & 0.065 \\
\hline
\end{tabular}

Notes: SPT\# = results with hearing aids only; SPT@= without hearing aids before and with hearing aids after 3 months.

Abbreviations: AV, Aversiveness of sound; BN, Background Noise; EC, Ease of Communication; RV, Reverberation of sound; SPT, speech perception test. 

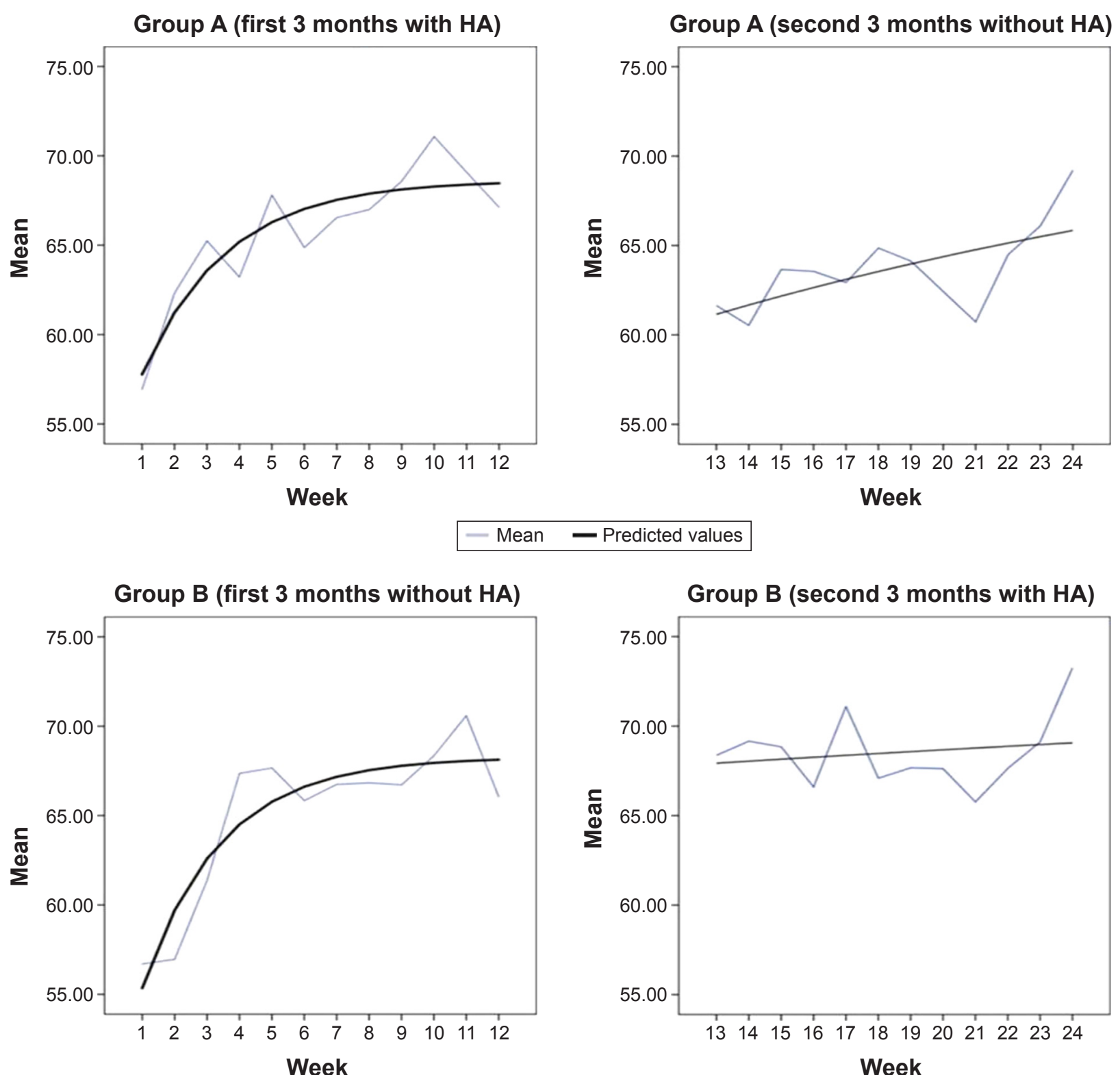

- Predicted values

\section{Week}

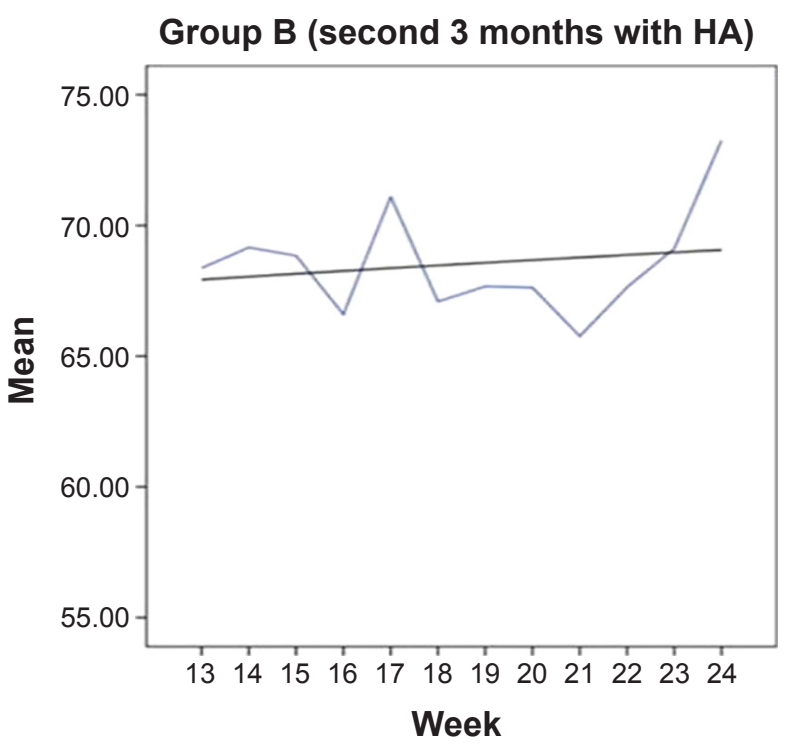

- Mean - Predicted values

Figure 2 Experimental data and learning curves derived from speech tracking sessions for groups $A$ and $B$.

Abbreviation: HA, hearing aid.

that forgetting was slower. However, Figure 2 shows similar learning and forgetting rates for Group A and Group B in the first 3 months. For the second 6 months, there was an initial decline for Group A when hearing aids were removed, but this decline was quickly reversed, with similar endpoints achieved for groups A and B.

\section{Crossover analysis for the effects of hearing aids and auditory training}

After the 6-month follow-up period, 9 (22.5\%) out of 40 participants withdrew from the study for the following reasons: discomfort after wearing the hearing aids, health issues, personal reasons and inability to attend weekly auditory training sessions. Overall, 17 (85\%) participants from Group A and 14 (70\%) participants from Group B completed all measures of the study from baseline to 6 months.

The mixed model analysis was, therefore, completed only for participants who did not withdraw from the study, in order to determine whether the hearing aids had a significant effect on cognition, the APHAB measures and the psychosocial measures. In addition, we tested for significant changes between the 3 and 6 months assessments, while controlling 
for baseline levels. The carryover effect was designed to detect any treatment order effect associated with the hearing aid usage.

We found significant improvements in depressive symptoms from 3 to 6 months with a moderate to large effect size (Cohen's $d=0.87$ ). In addition, we found a significant deterioration in Aversiveness of sound when hearing aids were worn. A significant carryover effect for delayed recognition memory was also found, invalidating the results for this cognition measure (see Table S4 for results of the mixed model crossover analysis).

\section{Discussion}

In this pilot study, we demonstrated the relationship between cognition and speech perception. This confirms the results of several empirical studies which have successfully established the link between cognitive abilities and speech recognition performance in first-time hearing aid users. ${ }^{27}$ However, only one direct cognition relationship with hearing loss was found, despite a strong relationship between hearing loss and speech perception. The significant correlation between cognition, as measured by the Incongruent Stroop test, and hearing loss needs further investigation. This result may suggest that tests of visual Incongruent Stroop capability could be an important addition to aural rehabilitative assessments. ${ }^{28}$

Second, hearing aid use was associated with improved speech perception, increasing the audibility of sounds. Increases in Aversiveness were also detected, but this was expected. ${ }^{29}$ Studies have shown that depression is associated with hearing impairment, ${ }^{30}$ and in this pilot study, we found significant improvement in depressive symptoms over the course of the study. This result is consistent with previous studies suggesting that the short-term use of hearing aids reduces depression. ${ }^{6,31}$ Depression is both a risk factor and a prodromal of Alzheimer's disease and is a common occurrence in all types of dementias as well as in mild cognitive impairment. ${ }^{32}$ Having depression reduces the quality of life, exacerbates cognitive and functional impairment and is associated with increased mortality. ${ }^{33}$ Therefore, our findings suggest that management of hearing loss could improve the life conditions of adults and may reduce the burden associated with dementia.

Third, no evidence was found to suggest that it is easier to learn to do speech tracking when participants use hearing aids than when they do not. Also, data depicted in Figure 2 are similar for both hearing aid users (Group A) and listeners without hearing aids (Group B) during the first 3 months of the auditory training program. This is an unexpected result requiring further investigation. This could imply that, in the first 3 months, all study participants were learning how to do auditory training rather than learning to improve their understanding of speech. Alternatively, it could be that it was learning by the researcher/trainer about how to correct mistakes in the most efficient way during auditory training, which resulted in improved tracking rates for both groups.

Finally, there was no significant improvement in cognition and social interaction over a 6-month period. One interpretation of this result is that hearing treatment may take longer than 6 months to impact cognition.

A limitation of the study was that daily hours of hearing aid usage were not reliably assessed and could not, therefore, be included in the analysis. This was due to hardware and software issues in the data-logging function installed in the hearing aids. This means that it is impossible to determine to what extent participants actually made use of the hearing aids they were given, outside of their auditory training sessions. Also, this study was underpowered, given the small sample size and high attrition rate. Larger research studies, preferably taking brain function into account through neuroimaging, are therefore needed to establish whether any causal association between hearing aid use and cognitive performance exists.

\section{Conclusion}

Recognition of hearing loss as a risk factor for dementia is relatively new, and results of cohort studies have suggested that even mild levels of hearing loss increase the long-term risk of cognitive decline and dementia in individuals who are cognitively intact but hearing impaired at baseline. ${ }^{3,34,35}$ There was no improvement in cognition observed in this study despite the usage of auditory training in addition to hearing aids. However, the baseline results clearly indicated better cognition performance in several domains in the case of participants with better speech perception. The effects of auditory interventions on depression over a 6-month period also showed significant effects in this study.

Given the limitations of this study, including the small sample size, the magnitude of the effects reported here should not be interpreted as would be the case for a fully powered trial. ${ }^{36}$ Our baseline results have provided the motivation needed to proceed with a full-scale, randomized hearing loss intervention and a longer neuroimaging study with cognitive outcomes measured in the short term as well as after several years hearing aid use is needed. ${ }^{37}$ This may be the first prospective cohort randomized controlled trial to test the neural, cognitive and psychosocial efficacy of hearing aid use in adults with post-lingual SNHL. 
In view of the problems encountered with the extraction of logged hearing aid use data in this pilot study, future studies will ensure that participants are instructed to manually record hours of hearing aid use every week, in order to confirm the automatically logged hearing use records. It is also hoped that our planned hearing loss intervention study, which will investigate the long-term impact of hearing aids on cognitive function, ${ }^{37}$ will address a further limitation of this pilot study.

\section{Data sharing statement}

Following publication, the authors intend to share all of the individual participant de-identified data collected that underlie the results reported in this article, upon request. The de-identified data will be shared with investigators whose proposed use of data has been approved by an independent review committee identified for this purpose. The study protocol will also be made available on request.

\section{Acknowledgments}

We are grateful to all the clinicians at Blamey and Saunders Hearing Pty Ltd and the retirement aged care facilities in Melbourne who participated in this research study. We would also like to acknowledge Professor Frank R Lin, Professor Sunil Bhar, and Professor Peter Blamey for making substantial contributions to the work reported in this manuscript. In addition, we wish to thank Greg Kennedy for his guidance in terms of the Swinburne University Computerized Cognitive Assessment Battery testing.

\section{Author contributions}

All authors contributed toward data analysis, drafting and critically revising the paper, gave final approval of the version to be published, and agreed to be accountable for all aspects of the work.

\section{Disclosure}

The authors report no conflicts of interest in this work.

\section{References}

1. Lin FR. Hearing loss and cognition among older adults in the United States. J Gerontol A Biol Sci Med Sci. 2011;66A(10):1131-1136.

2. Meister H, Schreitmüller S, Grugel L, Beutner D, Walger M, Meister I. Examining speech perception in noise and cognitive functions in the elderly. Am J Audiol. 2013;22(2):310-312.

3. Lin FR, Metter EJ, O'Brien RJ, Resnick SM, Zonderman AB, Ferrucci L. Hearing loss and incident dementia. Arch Neurol. 2011;68(2):214-220.

4. Lin FR, Albert M. Hearing loss and dementia - who is listening? Aging Ment Health. 2014;18(6):671-673.

5. Campbell J, Sharma A. Compensatory changes in cortical resource allocation in adults with hearing loss. Front Syst Neurosci. 2013;7:71.
6. Acar B, Yurekli MF, Babademez MA, Karabulut H, Karasen RM. Effects of hearing aids on cognitive functions and depressive signs in elderly people. Arch Gerontol Geriatr. 2011;52(3):250-252.

7. Ferguson M, Brandreth M, Brassington W, Leighton P, Wharrad H. A randomized controlled trial to evaluate the benefits of a multimedia educational program for first-time hearing aid users. Ear Hear. 2016; 37(2):123-136.

8. Chien W, Lin FR. Prevalence of hearing aid use among older adults in the United States. Arch Intern Med. 2012;172(3):292-293.

9. Meyer C, Hickson L. What factors influence help-seeking for hearing impairment and hearing aid adoption in older adults? Int $J$ Audiol. 2012;51(2):66-74

10. Olson AD. Options for auditory training for adults with hearing loss. Semin Hear. 2015;36(4):284-295.

11. Valentijn SA, van Boxtel MP, van Hooren SA, et al. Change in sensory functioning predicts change in cognitive functioning: results from a 6-year follow-up in the Maastricht Aging Study. J Am Geriatr Soc. 2005;53(3):374-380.

12. Blamey PJ, Alcantara JI. (1994). Research in auditory training. In J. Gagne \& N. Tye-Murray (Ed.), Research in audiological rehabilitation: current trends and future directions (pp. 161-191). Cedar Falls, Iowa: Academy of Rehabilitative Audiology.

13. Sweetow R, Palmer CV. Efficacy of individual auditory training in adults: a systematic review of the evidence. J Am Acad Audiol. 2005;16(7): 494-504.

14. Humes LE, Burk MH, Strauser LE, Kinney DL. Development and efficacy of a frequent-word auditory training protocol for older adults with impaired hearing. Ear Hear. 2009;30(5):613-627.

15. Nkyekyer J, Meyer D, Blamey PJ, Pipingas A, Bhar S. Investigating the impact of hearing aid use and auditory training on cognition, depressive symptoms, and social interaction in adults with hearing loss: protocol for a crossover trial. JMIR Res Protoc. 2018;7(3):e85.

16. World Medical Association. World Medical Association Declaration of Helsinki. Ethical principles for medical research involving human subjects. Bull World Health Organ. 2001;79(4):373.

17. de Filippo CL, Scott BL. A method for training and evaluating the reception of ongoing speech. J Acoust Soc Am. 1978;63(4): 1186-1192.

18. Blamey PJ, Blamey JK, Saunders E. Effectiveness of a teleaudiology approach to hearing aid fitting. J Telemed Telecare. 2015;21(8):474-478.

19. Blamey P, Saunders E. Predicting speech perception from the audiogram and vice versa. CAA Canadian Audiologist. 2018;2(1). Available from: http://www.canadianaudiologist.ca/issue/volume-2-issue-1-2015/ predicting-speech-perception-from-the-audiogram-and-vice-versa/. Accessed December 21, 2018.

20. Henry BA, McDermott HJ, McKay CM, James CJ, Clark GM. A frequency importance function for a new monosyllabic word test. Scientific publications, vol. 11, 1998-1999, no. 1116. 1998. Available from:https://minerva-access.unimelb.edu.au/bitstream/handle/11343/27518/119500_vol11_1116.pdf?sequence=1\&isAllowed=y. Accessed December 21, 2018.

21. Peterson GE, Lehiste I. Revised CNC lists for auditory tests. J Speech Hear Disord. 1962;27(1):62-70.

22. Cox RM, Alexander GC. The abbreviated profile of hearing aid benefit. Ear Hear. 1995;16(2):176-186.

23. Pipingas A, Harris E, Tournier E, King R, Kras M, Stough CK. Assessing the efficacy of nutraceutical interventions on cognitive functioning in the elderly. Curr Top Nutraceutical Res. 2010;8(2/3):79-88.

24. Burke WJ, Roccaforte WH, Wengel SP. The short form of the Geriatric Depression Scale: a comparison with the 30-item form. J Geriatr Psychiatry Neurol. 1991;4(3):173-178.

25. Yesavage JA, Sheikh JI. 9/Geriatric depression scale (GDS) recent evidence and development of a shorter version. Clin Gerontol. 1986; 5(1-2):165-173.

26. Berkman LF, Syme SL. Social networks, host resistance, and mortality: a nine-year follow-up study of Alameda County residents. Am J Epidemiol. 1979;109(2):186-204. 
27. Lunner T. Cognitive function in relation to hearing aid use. Int $J$ Audiol. 2003;42(Suppl 1):49-58.

28. Roberts KL, Hall DA. Examining a supramodal network for conflict processing: a systematic review and novel functional magnetic resonance imaging data for related visual and auditory Stroop tasks. $J \operatorname{Cog} n$ Neurosci. 2008;20(6):1063-1078.

29. Blamey P, Martin LF, Saunders E. Hearing Aid Benefit as a Function of Hearing Loss. Melbourne, Australia: Australian Speech Science and Technology Association; 2010.

30. Strawbridge WJ, Wallhagen MI, Shema SJ, Kaplan GA. Negative consequences of hearing impairment in old age: a longitudinal analysis. Gerontologist. 2000;40(3):320-326.

31. Boi R, Racca L, Cavallero A, et al. Hearing loss and depressive symptoms in elderly patients. Geriatr Gerontol Int. 2012;12(3):440-445.
32. Enache D, Winblad B, Aarsland D. Depression in dementia: epidemiology, mechanisms, and treatment. Curr Opin Psychiatry. 2011;24(6):461-472.

33. Greenwald BS, Kramer-Ginsberg E, Marin DB, et al. Dementia with coexistent major depression. Am J Psychiatry. 1989;146(11):1472.

34. Livingston G, Sommerlad A, Orgeta V, et al. Dementia prevention, intervention, and care. The Lancet. 2017;390(10113):2673-2734.

35. Deal JA, Betz J, Yaffe K. Hearing impairment and incident dementia and cognitive decline in older adults: the health $\mathrm{ABC}$ study. J Gerontol A Biol Sci Med Sci. 2017;72(5):703-709.

36. Leon AC, Davis LL, Kraemer HC. The role and interpretation of pilot studies in clinical research. J Psychiatr Res. 2011;45(5):626-629.

37. Hughes ME, Nkyekyer J, Innes-Brown H, et al. Hearing aid use in older adults with postlingual sensorineural hearing loss: protocol for a prospective cohort study. JMIR Res Protoc. 2018;7(10):e174. 


\section{Supplementary materials}

Table S3 suggests that forgetting and learning rates were significant only for the first 3 months for both groups.

In Table S4, in addition to controlling for baseline outcome measures, we controlled for baseline scores for Contextual Working Memory and SQRT (Aversiveness) because there were large differences between the two groups in these variables at baseline. In addition, age and attrition probability were controlled for, in order to adjust for age effects and any attrition bias. In these analyses, effect sizes (Cohen's $d$ ) were obtained by dividing the estimated coefficients by the residual SD, as recommended by Feingold.

Confirming the results from Table 1, there was a significant increase in Aversiveness when a hearing aid was worn, with marginal mean values of 4.431 and 5.781 for the two conditions (Cohen's $d=-1.01$ ). There was a significant decline in delayed recognition memory performance when hearing aids were used (Cohen's $d=0.95$ ). There was also a narrowly significant carryover effect in the case of delayed recognition memory.

Table SI Baseline characteristics

\begin{tabular}{|c|c|c|c|c|c|c|}
\hline Characteristics & $\begin{array}{l}\text { Group A } \\
(n=20)\end{array}$ & $\begin{array}{l}\text { Group B } \\
(n=20)\end{array}$ & $\begin{array}{l}\text { Total } \\
(\mathbf{N}=40)\end{array}$ & $\begin{array}{l}\text { Test } \\
\text { statistics }\end{array}$ & $P$-value & Cohen's d \\
\hline $\begin{array}{l}\text { Gender, n (\%) } \\
\text { Male } \\
\text { Female }\end{array}$ & $\begin{array}{l}8(40.0) \\
12(60.0)\end{array}$ & $\begin{array}{l}\text { II (55.0) } \\
9(45.0)\end{array}$ & $\begin{array}{l}19(47.5) \\
21(52.5)\end{array}$ & $\chi^{2}(I)=0.902$ & 0.342 & \\
\hline Age, mean (SD) & $75.9(7.9)$ & $76.5(7.5)$ & $76.2(7.6)$ & & 0.807 & -0.08 \\
\hline MMSE, mean (SD) & $28.4(0.7)$ & $28.5(0.9)$ & $28.5(0.8)$ & & 0.703 & -0.12 \\
\hline $\begin{array}{l}\text { Employment status, n (\%) } \\
\text { Employed } \\
\text { Retired }\end{array}$ & $\begin{array}{l}2(10.0) \\
18(90.0)\end{array}$ & $\begin{array}{l}5(25.0) \\
15(75.0)\end{array}$ & $\begin{array}{l}7(17.5) \\
33(82.5)\end{array}$ & $\chi^{2}(3)=3.273$ & 0.351 & \\
\hline $\begin{array}{l}\text { Education, } \mathrm{n}(\%) \\
\text { Primary/secondary/TAFE } \\
\text { University qualification }\end{array}$ & $\begin{array}{l}14(70.0) \\
6(30.0)\end{array}$ & $\begin{array}{l}13(65.0) \\
7(35.0)\end{array}$ & $\begin{array}{l}27(67.5) \\
13(32.5)\end{array}$ & $\chi^{2}(7)=4.254$ & 0.750 & \\
\hline $\begin{array}{l}\text { Hearing status, } \mathrm{n}(\%) \\
\text { Reported hearing trouble } \\
\text { Reported perceived tinnitus }\end{array}$ & $\begin{array}{l}18(90.0) \\
9(45.0) \\
\end{array}$ & $\begin{array}{l}17(85.0) \\
5(25.0)\end{array}$ & $\begin{array}{l}35(87.5) \\
14(35.0)\end{array}$ & $\begin{array}{l}\chi^{2}(1)=0.229 \\
\chi^{2}(3)=3.788\end{array}$ & $\begin{array}{l}0.633 \\
0.285 \\
\end{array}$ & \\
\hline Hearing loss better ear, mean (SD) & $37.6(7.6)$ & $39.5(11.3)$ & $38.5(9.6)$ & & 0.537 & -0.20 \\
\hline Speech perception test, mean (SD) & $119.5(18.1)$ & $111.2(22.0)$ & $115.4(20.3)$ & & 0.200 & 0.41 \\
\hline \multicolumn{7}{|c|}{ SUCCAB performance cognition measures, mean (SD) } \\
\hline $\begin{array}{l}\text { Simple Reaction Time } \\
\text { Complex Reaction Time } \\
\text { Immediate Recognition Memory } \\
\text { Delayed Recognition Memory } \\
\text { Stroop Congruent } \\
\text { Stroop Incongruent } \\
\text { Spatial Working Memory } \\
\text { Contextual Recognition Memory }\end{array}$ & $\begin{array}{l}331.0(45.4) \\
204.9(31.0) \\
67.7(24.4) \\
64.8(21.0) \\
116.4(17.5) \\
87.1(29.1) \\
60.5(21.9) \\
73.3(23.9)\end{array}$ & $\begin{array}{l}333.6(47.1) \\
203.2(25.7) \\
65.2(18.7) \\
58.6(11.6) \\
112.2(19.5) \\
85.7(20.7) \\
54.0(16.6) \\
59.9(20.5)\end{array}$ & $\begin{array}{l}332.3(45.7) \\
204.1(28.1) \\
66.4(21.5) \\
61.7(17.0) \\
114.3(18.4) \\
86.4(24.0) \\
57.3(19.5) \\
66.6(23.0)\end{array}$ & & $\begin{array}{l}0.858 \\
0.850 \\
0.723 \\
0.257 \\
0.479 \\
0.867 \\
0.297 \\
0.065\end{array}$ & $\begin{array}{l}-0.06 \\
0.06 \\
0.12 \\
0.37 \\
0.23 \\
0.06 \\
0.33 \\
0.61\end{array}$ \\
\hline \multicolumn{7}{|l|}{ APHAB, mean (SD) } \\
\hline $\begin{array}{l}\text { Ease of Communication } \\
\text { Effects of Reverberation } \\
\text { Effects of Background Noise } \\
\text { Aversiveness }\end{array}$ & $\begin{array}{l}26.0(19.0) \\
34.2(12.9) \\
36.4(13.5) \\
35.4(22.5)\end{array}$ & $\begin{array}{l}26.2(20.2) \\
34.6(14.0) \\
34.7(17.0) \\
22.5(19.0)\end{array}$ & $\begin{array}{l}26.1(19.4) \\
34.4(13.3) \\
35.5(15.2) \\
29.0(25.6)\end{array}$ & & $\begin{array}{l}0.959^{a} \\
0.922 \\
0.717 \\
0.058^{a}\end{array}$ & $\begin{array}{l}0.00 \\
-0.03 \\
-0.11 \\
0.64\end{array}$ \\
\hline \multicolumn{7}{|l|}{ Psychosocial measures, mean (SD) } \\
\hline $\begin{array}{l}\text { Depression } \\
\text { Social interaction }\end{array}$ & $\begin{array}{l}1.7(1.8) \\
32.5(11.3)\end{array}$ & $\begin{array}{l}1.5(1.5) \\
32.6(12.6)\end{array}$ & $\begin{array}{l}1.6(1.6) \\
32.5(11.8)\end{array}$ & & $\begin{array}{l}0.660^{\mathrm{a}} \\
0.969\end{array}$ & $\begin{array}{l}0.01 \\
0.00\end{array}$ \\
\hline
\end{tabular}

Note: aSquare root transformation.

Abbreviations: APHAB, Abbreviated Profile of Hearing Aid Benefit; MMSE, mini-mental state examination; SUCCAB, Swinburne University Computerized Cognitive Assessment Battery; TAFE, Technical and Further Education. 
Table S2 Outcome of hearing aid benefit with respect to SPT results when HAs were first fitted and when HAs were removed

\begin{tabular}{|c|c|c|c|c|c|c|c|c|}
\hline \multirow[t]{2}{*}{$\begin{array}{l}\text { Time } \\
\text { period }\end{array}$} & \multicolumn{4}{|c|}{$\begin{array}{l}\text { Group } A \text {, mean (SD) } \\
\mathrm{n}=20 \text { at baseline and } N=19 \text { at } 3 \text { months }\end{array}$} & \multicolumn{4}{|c|}{$\begin{array}{l}\text { Group } B \text {, mean (SD) } \\
n=14 \text { at } 3 \text { and } 6 \text { months }\end{array}$} \\
\hline & Without HAs & With HAs & $t$-value & $P$-value & Without HAs & With HAs & $t$-value & $P$-value \\
\hline Baseline & $119.5(18.1)$ & $124.9(15.3)$ & 3.03 & 0.007 & & & & \\
\hline 3 months & $121.5(18.4)$ & $124.5(17.2)$ & 1.32 & 0.203 & $119.0(18.6)$ & $124.0(16.7)$ & 2.012 & 0.065 \\
\hline 6 months & & & & & $122.9(17.8)$ & $121.6(21.8)$ & 0.488 & 0.634 \\
\hline
\end{tabular}

Abbreviations: HA, hearing aid; SPT, speech perception test.

Table S3 Estimated parameters (standard errors) derived from speech tracking data

\begin{tabular}{|c|c|c|c|c|}
\hline Condition & Group A & & Group B & \\
\hline $\begin{array}{l}\text { Number of sessions } \\
\text { per week }\end{array}$ & First 3 months with $\mathrm{HA}$ & Second 3 months without HA & First 3 months without $\mathrm{HA}$ & Second 3 months with $\mathrm{HA}$ \\
\hline Constant $(T)$ & $-3.803(5.22)$ & $-4 \mid .78(56.45)$ & $-3.046(2.92)$ & $-136.14(174.6)$ \\
\hline Forgetting rate per week (f) & $0.384(0.212)$ & $0.04 I(0.13)$ & $0.412(0.253)$ & $0.018(0.210)$ \\
\hline Learning rate per week $(L)$ & $26.35 I(14.03)$ & $3.02(8.29)$ & $28.09(16.84)$ & $1.33(14.35)$ \\
\hline
\end{tabular}

Notes: Significant forgetting and learning rates in bold. Standard errors for these estimates were obtained using bootstrapped samples.

Abbreviation: HA, hearing aid.

Table S4 Mixed model crossover analysis

\begin{tabular}{|c|c|c|c|c|c|}
\hline \multirow[t]{2}{*}{ Outcome measure } & \multicolumn{2}{|l|}{ Time effect } & \multicolumn{2}{|l|}{ Hearing aid effect } & \multirow{2}{*}{$\begin{array}{l}\text { Carryover } \\
\text { effect HA }\end{array}$} \\
\hline & $\begin{array}{l}\text { Coefficient } \\
\text { (standard error) }\end{array}$ & Cohen's d & $\begin{array}{l}\text { Coefficient } \\
\text { (standard error) }\end{array}$ & Cohen's d & \\
\hline Hearing loss & $-1.493(2.034)$ & -0.30 & $2.660(2.002)$ & 0.54 & $3.27 \mid(3.747)$ \\
\hline Speech perception test (woHA) & $3.423(3.301)$ & 0.43 & $-0.738(3.244)$ & -0.09 & $-4.018(6.069)$ \\
\hline \multicolumn{6}{|c|}{ SUCCAB performance cognition measures } \\
\hline Simple Reaction Time & 2.449 (I4.342) & 0.07 & $-9.656(14.141)$ & -0.28 & $-13.768(26.614)$ \\
\hline Complex Reaction Time & $-1.599(7.483)$ & -0.09 & $-7.833(7.390)$ & -0.45 & $-4.000(14.090)$ \\
\hline Immediate Recognition Memory & $-1.675(5.606)$ & -0.13 & $-7.469(5.522)$ & -0.58 & $-6.958(10.348)$ \\
\hline Delayed Recognition Memory & $6.337(5.714)$ & 0.47 & $-12.993(5.632)^{*}$ & -0.95 & $-22.280(10.190)^{*}$ \\
\hline Stroop Congruent & I.95I (4.80I) & 0.17 & $-4.989(4.683)$ & -0.44 & $-7.804(8.598)$ \\
\hline Stroop Incongruent & $6.057(8.201)$ & 0.35 & $-9.109(8.138)$ & -0.52 & $-11.565(15.800)$ \\
\hline Spatial Working Memory & $-5.38 \mathrm{I}(4.86 \mathrm{I})$ & -0.44 & $0.179(4.739)$ & 0.01 & $-I .111$ (7.076) \\
\hline Contextual Recognition Memory & $3.41 \mathrm{I}(5.072)$ & 0.27 & $0.153(4.953)$ & 0.01 & $-4.869(8.622)$ \\
\hline \multicolumn{6}{|l|}{ APHAB without $\mathrm{HA}$} \\
\hline SQRT (Ease of Communication) & $0.077(0.480)$ & 0.07 & $0.065(0.476)$ & 0.06 & $0.084(0.900)$ \\
\hline Reverberation & $-0.951(5.169)$ & -0.08 & I.448 (5.075) & 0.12 & $6.527(9.243)$ \\
\hline Background Noise & $2.957(5.272)$ & 0.23 & $-0.780(5.199)$ & -0.06 & $-1.495(9.807)$ \\
\hline SQRT (Aversiveness) & $-0.472(0.558)$ & -0.34 & $\mathrm{I} .387(0.547)^{*}$ & 1.01 & $\mathrm{I} .432(0.987)$ \\
\hline \multicolumn{6}{|l|}{ Psychosocial measures } \\
\hline SQRT (depression) & $-0.469(0.230)^{*}$ & -0.87 & $-0.090(0.226)$ & -0.17 & $0.056(0.403)$ \\
\hline Social interaction & $-1.489(3.26 \mathrm{I})$ & -0.19 & $-1.762(3.146)$ & -0.06 & $0.506(5.238)$ \\
\hline
\end{tabular}

Note: $* p<0.05$.

Abbreviations: APHAB, Abbreviated Profile of Hearing Aid Benefit; HA, hearing aid; SQRT, square root transformation; SUCCAB, Swinburne University Computerized Cognitive Assessment Battery; woHA, without hearing aids.

\section{Reference}

1. Feingold A. A regression framework for effect size assessments in longitudinal modeling of group differences. Rev Gen Psychol. 2013;17(1): $111-121$. 
Clinical Interventions in Aging

\section{Publish your work in this journal}

Clinical Interventions in Aging is an international, peer-reviewed journal focusing on evidence-based reports on the value or lack thereof of treatments intended to prevent or delay the onset of maladaptive correlates of aging in human beings. This journal is indexed on PubMed Central, MedLine,

CAS, Scopus and the Elsevier Bibliographic databases. The manuscript management system is completely online and includes a very quick and fair peer-review system, which is all easy to use. Visit http://www.dovepress. $\mathrm{com} /$ testimonials.php to read real quotes from published authors.

Submit your manuscript here: http://www.dovepress.com/clinical-interventions-in-aging-journal 